PROCEEDINGS OF THE

AMERICAN MATHEMATICAL SOCIETY

Volume 136, Number 1, January 2008, Pages 1-9

S 0002-9939(07)08840-5

Article electronically published on October 11, 2007

\title{
A CALABI-YAU THREEFOLD WITH BRAUER GROUP $(\mathbb{Z} / 8 \mathbb{Z})^{2}$
}

\author{
MARK GROSS AND SIMONE PAVANELLI \\ (Communicated by Michael Stillman)
}

\begin{abstract}
We compute the Brauer group of a Calabi-Yau threefold discovered by the first author and Sorin Popescu, and find it is $(\mathbb{Z} / 8 \mathbb{Z})^{2}$, the largest known Brauer group of a non-singular Calabi-Yau threefold.
\end{abstract}

\section{INTRODUCTION}

If $X$ is a non-singular Calabi-Yau threefold with $b_{1}(X)=0$, then the analytic cohomological Brauer group $H_{a n}^{2}\left(X, \mathcal{O}_{X}^{*}\right)$ is isomorphic to $H^{3}(X, \mathbb{Z})_{\text {tors }} \cong$ $H^{4}(X, \mathbb{Z})_{\text {tors }} \cong H_{2}(X, \mathbb{Z})_{\text {tors }}$ (and this coincides with the cohomological Brauer group in the étale topology). There has been recent interest in finding examples of Calabi-Yau threefolds with non-trivial Brauer group motivated partly by string theory ([3]), partly by their role in mirror symmetry ([3], [12]), and partly by the study of twisted derived categories ([7, 8]). For a long time, there seemed to be no examples of non-trivial Brauer groups for simply connected, non-singular CalabiYau threefolds. Recent work of Batyrev and Kreuzer 4] identified all Calabi-Yau hypersurfaces in toric varieties with non-trivial Brauer group, and examples were found with groups of orders 2,3 , and 5 . In this note we study a Calabi-Yau threefold first discovered by the first author and Sorin Popescu in [15], and show that it has Brauer group $(\mathbb{Z} / 8 \mathbb{Z})^{2}$.

This Calabi-Yau threefold was studied extensively in the second author's thesis [17, motivated by the hope that it would be self-mirror. Carrying out the $A$ and $B$ model instanton calculations provided supporting evidence for this idea; however, a deeper analysis using the Strominger-Yau-Zaslow conjecture led to the expectation that the mirror was not simply connected. These results could be explained if the original Calabi-Yau had a non-trivial Brauer group, and is the universal cover of its mirror. While this has not yet been proven, this proposal led to the calculation of the Brauer group presented here.

Unfortunately, calculating torsion groups of cohomology is often difficult, and we apologize if the proof given here is unnecessarily complicated.

\section{THE EXAMPLE}

We recall the construction of a Calabi-Yau threefold $V_{8, y} \subseteq \mathbb{P}^{7}$ from [15, $\S 6$. Here $y$ is a parameter lying in a projective plane we call $\mathbb{P}_{-}^{2} \subseteq \mathbb{P}^{7}$ given by the

Received by the editors December 14, 2005 and, in revised form, July 10, 2006.

2000 Mathematics Subject Classification. Primary 14J32.

This work was partially supported by NSF grant 0204326 and 0505325 .

(C)2007 American Mathematical Society 
equations

$$
x_{0}=x_{1}+x_{7}=x_{2}+x_{6}=x_{3}+x_{5}=x_{4}=0 .
$$

Let $\sigma, \tau: k\left[x_{0}, \ldots, x_{7}\right] \rightarrow k\left[x_{0}, \ldots, x_{7}\right]$ be the automorphisms given by $\sigma\left(x_{i}\right)=x_{i-1}$ and $\tau\left(x_{i}\right)=\xi^{-i} x_{i}$, with indices taken modulo 8 and $\xi$ a primitive 8th root of unity. These automorphisms generate the Heisenberg group $\mathbb{H}_{8}$, a central extension of $(\mathbb{Z} / 8 \mathbb{Z})^{2}$ by $\mu_{8}$, and the action of $\mathbb{H}_{8}$ on $k\left[x_{0}, \ldots, x_{7}\right]$ induces an action of $\mathbb{H}_{8}$ on $\mathbb{P}^{7}$, which factors through the quotient $(\mathbb{Z} / 8 \mathbb{Z})^{2}$. Any abelian surface $A$ with a polarization of type $(1,8)$ can be mapped to $\mathbb{P}^{7}$ in such a way that the action of $\mathbb{H}_{8}$ on $\mathbb{P}^{7}$ induces translation on $A$ by elements of the kernel of the polarization. For a review of this standard theory, see $\S 1$ of [14].

Take

$$
\begin{aligned}
& f_{0}=x_{0}^{2}+x_{4}^{2}, \\
& f_{1}=x_{1} x_{7}+x_{3} x_{5}, \\
& f_{2}=x_{2} x_{6} .
\end{aligned}
$$

We write $y \in \mathbb{P}_{-}^{2}$ as $y=\left(y_{1}: y_{2}: y_{3}\right)$, by which we mean the point $\left(0: y_{1}: y_{2}: y_{3}\right.$ : $\left.0:-y_{3}:-y_{2}:-y_{1}\right)$. Given $y$, set

$$
f=y_{1} y_{3} f_{0}-y_{2}^{2} f_{1}+\left(y_{1}^{2}+y_{3}^{2}\right) f_{2}
$$

and define $V_{8, y}$ by the equations

$$
f=\sigma(f)=\sigma^{2}(f)=\sigma^{3}(f)=0 .
$$

Then we have

Theorem 1.1. Let $y \in \mathbb{P}_{-}^{2}$ be general.

(1) $V_{8, y}$ is a complete intersection, singular only at 64 points, each singularity being an ordinary double point. These 64 points are the $\mathbb{H}_{8}$-orbit of $y$.

(2) There is a small resolution $\mu: V_{8, y}^{1} \rightarrow V_{8, y}$ and a fibration $\pi: V_{8, y}^{1} \rightarrow \mathbb{P}^{1}$ whose general fibre is an abelian surface with a $(1,8)$ polarization induced by its embedding in $\mathbb{P}^{7}$. (In [15], this map is called $\left.\pi_{1}\right)$.

(3) $\chi\left(V_{8, y}^{1}\right)=0$ and $h^{1,1}\left(V_{8, y}^{1}\right)=h^{1,2}\left(V_{8, y}^{1}\right)=2$.

(4) $H^{2}\left(V_{8, y}^{1}, \mathbb{Z}\right) /$ Tors is generated by $H$, the pull-back of the class of a hyperplane section of $V_{8, y}$, and $A$ the class of a fibre of $\pi$. Also $H^{4}\left(V_{8, y}^{1}, \mathbb{Z}\right) /$ Tors is generated by $[l]$, the class of a line in $V_{8, y}$ disjoint from $\operatorname{Sing}\left(V_{8, y}\right)$, and $[e]$ the class of an exceptional curve of $\mu$. We have A.e $=1$, so any exceptional curve of $\mu$ is a section of $\pi$. In addition,

$$
H^{3}=16, H^{2} A=16, A^{2}=0 .
$$

(5) $V_{8, y} \cap \mathbb{P}_{-}^{2}=\left\{y, \sigma^{4}(y), \tau^{4}(y), \sigma^{4} \tau^{4}(y)\right\}$, and $V_{8, y}$ contains every $\mathbb{H}_{8}$-invariant abelian surface $A \subseteq \mathbb{P}^{7}$ with $y \in A$ that is embedded with a polarization of type $(1,8)$. The proper transform of each such abelian surface in $V_{8, y}^{1}$ is a fibre of $\pi$.

(6) Any $\mathbb{H}_{8}$-invariant abelian surface $A \subseteq \mathbb{P}^{7}$ embedded with a polarization of type $(1,8)$ intersects $\mathbb{P}_{-}^{2}$ in four points.

Proof. This is Theorems 6.5, 6.7, 6.9 and Remark 6.10 of [15].

Lemma 1.2. Let $V_{8, y}^{1, \#}=V_{8, y}^{1} \backslash \operatorname{Crit}(\pi)$, where $\operatorname{Crit}(\pi)$ denotes the closed subset where $\pi$ is not smooth. Then after fixing any one of the 64 exceptional curves of $\mu$ to be the identity section of $\pi, \pi^{\#}: V_{8, y}^{1, \#} \rightarrow B:=\mathbb{P}^{1}$ is a group scheme. 
Proof. We will in fact show that $\pi^{\#}$ is a Néron model, hence a group scheme.

We first note that no fibre of $\pi$ can be reducible or non-reduced: by Theorem 1.1. (4), any surface $S \subseteq V_{8, y}^{1}$ has $\operatorname{deg} \mu(S)$ divisible by 16 . Since $\operatorname{deg} \mu(A)=16, A$ cannot be written as the sum of other effective divisors. Thus each singular fibre is at worst singular along a curve.

Working locally at a point $b \in B$, we note that given a local section $\sigma$ of $\pi$ over an étale neighbourhood $U$ of $b$, translation by this section induces a birational map $T_{\sigma}: V_{U^{--} \rightarrow V_{U}}$, where $V_{U}=V_{8, y}^{1} \times_{B} U$. However, $\pi_{U}: V_{U} \rightarrow U$ is relatively minimal, and hence $T_{\sigma}$ becomes an isomorphism after performing a sequence of flops on $V_{U}$ over $U$. On the other hand, the general fibre of $\pi$ has Picard number 1 and every fibre is irreducible, from which it follows that the relative $N^{1}\left(V_{U} / U\right)$ is one-dimensional. Here $N^{1}\left(V_{U} / U\right)=\left(\operatorname{Pic} V_{U} \otimes \mathbb{R}\right) / \equiv$, where $D \equiv 0$ if $D . C=0$ for all curves $C$ contracted by $\pi$. This implies that if any curve in a fibre of $\pi_{U}$ is contracted, all curves are, and there are no curves in fibres of $\pi_{U}$ which can be flopped. Thus $T_{\sigma}$ is biregular.

Given this, one can now adapt the standard proof that a relatively minimal elliptic fibration over a curve is a Néron model after removing non-smooth points; see for example [6, page 21, Prop. 1, or 2], Prop. 1.15, page 218.

Let $E \subseteq \mathbb{P}^{7}$ be an elliptic normal curve (of degree 8). Then a translation scroll over $E$ is obtained by choosing a point $\rho \in E$, and taking

$$
S_{E, \rho}:=\bigcup_{P \in E}\langle P, P+\rho\rangle,
$$

where $\langle P, P+\rho\rangle$ denotes the line joining $P$ and $P+\rho$. For general choice of $\rho$, the translation scroll is singular only along $E$.

Proposition 1.3. For general choice of $y, \pi$ has eight singular fibres, and they are all translation scrolls.

Proof. Consider

$$
0 \rightarrow \pi^{*} \Omega_{\mathbb{P}^{1}}^{1} \rightarrow \Omega_{V_{8, y}^{1}}^{1} \rightarrow \Omega_{V_{8, y}^{1} / \mathbb{P}^{1}}^{1} \rightarrow 0 .
$$

Dualizing this gives the exact sequence

$$
0 \longrightarrow\left(\Omega_{V_{8, y}^{1} / \mathbb{P}^{1}}^{1}\right)^{\vee} \stackrel{\alpha}{\longrightarrow} \mathcal{T}_{V_{8, y}^{1}} \stackrel{\pi_{*}}{\longrightarrow} \pi^{*} \mathcal{T}_{\mathbb{P}^{1}} .
$$

Clearly $\pi_{*}$ drops rank precisely on $\operatorname{Crit}(\pi)$. Choosing an exceptional curve $e$ as the zero-section of $\pi$ as in Lemma 1.2, identifying $e$ with $\mathbb{P}^{1}$ via $\pi$, the fact that $\pi^{\#}$ is a group scheme shows that the relative tangent bundle $\mathcal{T}_{V_{8, y}^{1, \#} / \mathbb{P}^{1}}$ is isomorphic to $\pi^{\# *} \mathcal{N}_{e / V_{8, y}^{1}}$. But $\mathcal{T}_{V_{8, y}^{1, \#} / \mathbb{P}^{1}}=\left.\left(\Omega_{V_{8, y}^{1} / \mathbb{P}^{1}}^{1}\right)^{\vee}\right|_{V_{8, y}^{1, \#}}$ and $\left(\Omega_{V_{8, y}^{1} / \mathbb{P}^{1}}^{1}\right)^{\vee}$ is reflexive, and hence is equal to $j_{*}\left(\left.\left(\Omega_{V_{8, y}^{1} / \mathbb{P}^{1}}^{1}\right)^{\vee}\right|_{V_{8, y}^{1, \#}}\right)$, where $j: V_{8, y}^{1, \#} \rightarrow V_{8, y}^{1}$ is the inclusion. Thus the exact sequence (1) becomes

$$
0 \longrightarrow \pi^{*}\left(\mathcal{O}_{\mathbb{P}^{1}}(-1) \oplus \mathcal{O}_{\mathbb{P}^{1}}(-1)\right) \stackrel{\alpha}{\longrightarrow} \mathcal{T}_{V_{8, y}^{1}} \stackrel{\pi_{*}}{\longrightarrow} \pi^{*} \mathcal{O}_{\mathbb{P}^{1}}(2) .
$$

On the other hand, $\alpha$ must drop rank precisely when $\pi_{*}$ drops rank. Since this locus is at most dimension one, it follows this happens on a pure dimension one locus, and the class of this degeneracy locus is $c_{2}\left(\mathcal{T}_{V_{8, y}^{1}} \otimes \pi^{*}\left(\mathcal{O}_{\mathbb{P}^{1}}(1)\right)\right)=c_{2}\left(V_{8, y}^{1}\right)$. On the other hand, $\mu_{*} c_{2}\left(V_{8, y}^{1}\right)$ has degree equal to the degree of the second Chern class of a general $(2,2,2,2)$ complete intersection in $\mathbb{P}^{7}$. This degree is 64 , so we 
find $\operatorname{deg} \mu(\operatorname{Crit}(\pi)) \leq 64$ (with equality if the correct scheme structure is put on $\operatorname{Crit}(\pi))$.

We now show for general $y \in \mathbb{P}_{-}^{2}$, there are at least $8 \mathbb{H}_{8}$-invariant translation scrolls containing $y$. By [14], Theorem 3.1, any such translation scroll is a degeneration of $\mathbb{H}_{8}$-invariant abelian surfaces, and hence by Theorem 1.1, (5) and (6), is a fibre of $\pi$. Since each translation scroll is singular along an elliptic curve of degree 8 , this will demonstrate that $V_{8, y}^{1}$ has precisely 8 singular fibres, each a translation scroll.

Consider the $4 \times 4$ Moore matrix

$$
M_{4}=\left(x_{i+j} y_{i-j}+x_{i+j+4} y_{i-j+4}\right)_{0 \leq i, j \leq 3} .
$$

Here, all indices are modulo 8. Then by [14, Cor. 2.2, if $E \subseteq \mathbb{P}^{7}$ is an $\mathbb{H}_{8}$-invariant elliptic curve, then $M_{4}$ is rank one on $E \times E \subseteq \mathbb{P}^{7} \times \mathbb{P}^{7}$. This shows in particular that if $S(8)$ denotes the surface which is the closure of the union of all $\mathbb{H}_{8}$-invariant elliptic normal curves of degree 8 in $\mathbb{P}^{7}$, then the rank of the matrix

$$
\left(y_{i+j} y_{i-j}+y_{i+j+4} y_{i-j+4}\right)_{0 \leq i, j \leq 3}
$$

is one on $S(8)$. The compactified moduli space of $\mathbb{H}_{8}$-invariant elliptic curves is the compactified moduli space of elliptic curves with a full level structure of degree 8. (A full level structure is an identification of the 8-torsion points on $E$ with $(\mathbb{Z} / 8 \mathbb{Z})^{2}$.) This space is the modular curve $X(8)$; by [18, $\S 1.6$, the genus of $X(8)$ is 5 . (We remark that $S(8)$ is not the universal family over $X(8)$ because it does not have a well-defined section; rather it is a twist of the universal family.)

Next, given a general $E \subseteq \mathbb{P}^{7}$ an $\mathbb{H}_{8}$-invariant elliptic normal curve, the union of translation scrolls of $E$ is the secant variety $\operatorname{Sec}(E)$, whose ideal is generated by the $3 \times 3$ minors of $M_{4}$ whenever $y$ is chosen to be a general point of $E$. This is 14, Theorem 5.2. Let us consider the intersection of $\operatorname{Sec}(E)$ with $\mathbb{P}_{-}^{2}$ : this is obtained by setting $x_{i}=-x_{8-i}$ in the Moore matrix, and $\operatorname{Sec}(E) \cap \mathbb{P}_{-}^{2}$ is then the locus where the $3 \times 3$ minors of this latter matrix vanish. This matrix is

$$
\left(\begin{array}{cccc}
0 & -x_{3} y_{3}+x_{1} y_{7} & -x_{2} y_{2}+x_{2} y_{6} & -x_{1} y_{1}+x_{3} y_{5} \\
x_{1} y_{1}-x_{3} y_{5} & x_{2} y_{0}-x_{2} y_{4} & -x_{1} y_{3}+x_{3} y_{7} & 0 \\
x_{2} y_{2}-x_{2} y_{6} & x_{3} y_{1}-x_{1} y_{5} & 0 & x_{1} y_{3}-x_{3} y_{7} \\
x_{3} y_{3}-x_{1} y_{7} & 0 & -x_{3} y_{1}+x_{1} y_{5} & -x_{2} y_{0}+x_{2} y_{4}
\end{array}\right) .
$$

We note that if we interchange the second and fourth rows of this matrix, it becomes skew-symmetric, and hence the $3 \times 3$ minors vanish exactly where the Pfaffian of this matrix vanishes. The Pfaffian can be computed as

$$
w_{0}\left(y_{1}^{2}-y_{3}^{2}+y_{5}^{2}-y_{7}^{2}\right) / 2+w_{1}\left(y_{0}-y_{4}\right)\left(y_{2}-y_{6}\right)+w_{2}\left(y_{3} y_{7}-y_{1} y_{5}\right)
$$

where

$$
w_{0}=2 x_{1} x_{3}, \quad w_{1}=-x_{2}^{2}, \quad w_{2}=x_{1}^{2}+x_{3}^{2} .
$$

Thus in particular, $\operatorname{Sec}(E) \cap \mathbb{P}_{-}^{2}$ is a conic (as $\operatorname{Sec}(E)$ cannot contain all of $\mathbb{P}_{-}^{2}$ ), and this gives a map $\psi^{\prime}$ from $X(8)$ to the space of conics in $\mathbb{P}_{-}^{2}$ : for $p \in X(8), E_{p}$ the corresponding $\mathbb{H}_{8}$-invariant elliptic curve in $\mathbb{P}^{7}, \psi^{\prime}(p)=\operatorname{Sec}\left(E_{p}\right) \cap \mathbb{P}_{-}^{2}$. More generally, the above equations show how a general point $y=\left(y_{0}: \cdots: y_{7}\right) \in \mathbb{P}^{7}$ determines a conic in $\mathbb{P}_{-}^{2}$, and hence define a rational map $\psi: \mathbb{P}^{7}-\rightarrow \mathbb{P}\left(H^{0}\left(\mathbb{P}_{-}^{2}, \mathcal{O}_{\mathbb{P}_{0}^{2}}(2)\right)\right)$ to the space of conics in $\mathbb{P}_{-}^{2}$. The image of this map lies in the $\mathbb{P}^{2}$ spanned by the conics $w_{0}, w_{1}$, and $w_{2}$. Let $\hat{w}_{0}, \hat{w}_{1}, \hat{w}_{2}$ be homogeneous coordinates on this $\mathbb{P}^{2}$, so $\psi^{*}\left(\hat{w}_{0}\right)=\left(y_{1}^{2}-y_{3}^{2}+y_{5}^{2}-y_{7}^{2}\right) / 2$ and so on. A simple calculation performed on 
Macaulay or Macaulay 2 ([ [] or [0] $)$ shows that $\psi^{*}\left(\hat{w}_{1}^{4}-8 \hat{w}_{0}^{3} \hat{w}_{2}-8 \hat{w}_{0} \hat{w}_{2}^{3}\right)$ is contained in the ideal generated by the $2 \times 2$ minors of $M_{4}(y, y)$. Thus in particular, $\psi(S(8))$ is contained in the curve $C$ defined by $\hat{w}_{1}^{4}-8 \hat{w}_{0}^{3} \hat{w}_{2}-8 \hat{w}_{0} \hat{w}_{2}^{3}=0$. This shows that $\psi^{\prime}$ factors through $C$. The map $\psi^{\prime}$ is non-constant, as was observed in the proof of [15], Theorem 6.3. As the genus of $C$ is 3 and the genus of $X(8)$ is 5, $\psi^{\prime}$ must be a double cover.

Thus, for $y \in \mathbb{P}_{-}^{2}$ general, there are precisely 8 points $p_{1}, \ldots, p_{8}$ of $X(8)$ such that $y \in \psi^{\prime}\left(p_{i}\right)$ for each $i$. For each such point $p_{i}$, there is at least one $\mathbb{H}_{8}$-invariant translation scroll $S_{E_{p_{i}}, \rho_{i}} \subseteq \operatorname{Sec}\left(E_{p_{i}}\right)$ containing $y$. This yields the desired 8 translation scrolls containing $y$.

We now calculate the Brauer group of $V_{8, y}^{1}$. We note that $\mathbb{H}_{8}$ acts on $V_{8, y}$ via the action on $\mathbb{P}^{7}$. It is easy to check this action is fixed-point free, and lifts to $V_{8, y}^{1}$, as it is given by translation by 8 -torsion sections on $V_{8, y}^{1}$. The center of $\mathbb{H}_{8}$ acts trivially on $\mathbb{P}^{7}$, hence acts trivially on $V_{8, y}^{1}$.

Theorem 1.4. $V_{8, y}^{1}$ is simply connected, $H^{4}\left(V_{8, y}^{1}, \mathbb{Z}\right)=\mathbb{Z}^{2} \oplus G$, where $G \cong \mathbb{H}_{8} / Z\left(\mathbb{H}_{8}\right)$ $\cong(\mathbb{Z} / 8 \mathbb{Z})^{2}$, and an inclusion $G \rightarrow H^{4}\left(V_{8, y}^{1}, \mathbb{Z}\right)$ is given by $\varphi \in G \mapsto\left[\varphi\left(e_{0}\right)\right]-\left[e_{0}\right]$, where $e_{0}$ is any one of the exceptional curves of $\mu$. Here $\varphi \in G$ acts via the action of $\mathbb{H}_{8}$ on $V_{8, y}^{1}$.

Proof. Let $Y$ be a non-singular $(2,2,2,2)$ complete intersection. Topologically, $Y$ is obtained from $V_{8, y}^{1}$ by replacing a neighbourhood diffeomorphic to $B^{4} \times S^{2}$ of each exceptional $S^{2}=\mathbb{P}^{1}$ with a neighbourhood diffeomorphic to $B^{3} \times S^{3}$. Here $B^{n}$ denotes an $n$-ball. In particular, each exceptional $S^{2}$ in $V_{8, y}^{1}$ is replaced by an $S^{3}$ in $Y$. Thus if $E$ is the union of exceptional two-spheres in $V_{8, y}^{1}, F \subseteq Y$ the union of three-spheres, then $V_{8, y}^{1} \backslash E$ is homeomorphic to $Y \backslash F$, and it follows from Van Kampen's theorem that $\pi_{1}\left(V_{8, y}^{1}\right) \cong \pi_{1}\left(V_{8, y}^{1} \backslash E\right) \cong \pi_{1}(Y)=0$.

In addition, the relative cohomology exact sequence yields sequences

$$
\begin{aligned}
0 & \rightarrow H^{3}\left(V_{8, y}^{1}, \mathbb{Z}\right) \rightarrow H^{3}\left(V_{8, y}^{1} \backslash E, \mathbb{Z}\right) \rightarrow H^{0}(E, \mathbb{Z}) \\
& \rightarrow \quad H^{4}\left(V_{8, y}^{1}, \mathbb{Z}\right) \rightarrow H^{4}\left(V_{8, y}^{1} \backslash E, \mathbb{Z}\right) \rightarrow H^{1}(E, \mathbb{Z})=0
\end{aligned}
$$

and

$$
0=H^{1}(F, \mathbb{Z}) \rightarrow H^{4}(Y, \mathbb{Z}) \rightarrow H^{4}(Y \backslash F, \mathbb{Z}) \rightarrow H^{2}(F, \mathbb{Z})=0 .
$$

Thus $\mathbb{Z} \cong H^{4}(Y, \mathbb{Z}) \cong H^{4}\left(V_{8, y}^{1}, \mathbb{Z}\right) / L$, where $L$ is the subgroup of $H^{4}\left(V_{8, y}^{1}, \mathbb{Z}\right)$ generated by Poincaré dual classes of exceptional curves. In particular, $H^{4}\left(V_{8, y}^{1}, \mathbb{Z}\right)_{\text {tors }}$ must be generated by classes $[e]-\left[e^{\prime}\right]$ with $e, e^{\prime}$ exceptional curves.

Now consider the Leray spectral sequence for $\pi: V_{8, y}^{1} \rightarrow B, B=\mathbb{P}^{1}$, with $E_{2}$ term $E_{2}^{p, q}=H^{p}\left(B, R^{q} \pi_{*} \mathbb{Z}\right)$. We have some work to do to get control over the various terms in this sequence.

We have $R^{p} \pi_{*} \mathbb{Z}=0$ for $p>4, \pi_{*} \mathbb{Z}=\mathbb{Z}$ and $R^{4} \pi_{*} \mathbb{Z}=\mathbb{Z}$, as all fibres are irreducible and have a well-defined orientation. Note also that if $B_{0}=B \backslash\{p \in$ $B \mid \pi^{-1}(p)$ is singular $\}, i: B_{0} \hookrightarrow B$ is the inclusion, and $\pi_{0}:\left.\pi\right|_{\pi^{-1}\left(B_{0}\right)}: \pi^{-1}\left(B_{0}\right) \rightarrow$ $B_{0}$, then

$$
i_{*} R^{p} \pi_{0 *} \mathbb{Z}=R^{p} \pi_{*} \mathbb{Z}
$$

for $0 \leq p \leq 4$. This is the notion of a simple torus fibration introduced in [11. To see this, note that from the standard construction of a smoothing of a translation scroll (see for example [1] or [16), a neighbourhood of the translation scroll in $X$ is topologically equivalent to $T^{2} \times X$, where $X \rightarrow D$ is a $T^{2}$-fibration over a disk 
with one degenerate fibre, a pinched torus (i.e. a Kodaira type $I_{1}$ fibre). Then in a suitable basis, the monodromy in the local system $R^{1} \pi_{0 *} \mathbb{Z}$ around this singular fibre is

$$
\left(\begin{array}{llll}
1 & 1 & 0 & 0 \\
0 & 1 & 0 & 0 \\
0 & 0 & 1 & 0 \\
0 & 0 & 0 & 1
\end{array}\right) .
$$

The statement about simplicity then follows as in [13, Theorem 1.3. In fact, it similarly follows that

$$
i_{*} R^{p} \pi_{0 *} \mathbb{Z} / n \mathbb{Z}=R^{p} \pi_{*} \mathbb{Z} / n \mathbb{Z}
$$

for $0 \leq p \leq 4$ and any integer $n$. We can now argue similarly to the proof of [12], Theorem 3.9, as follows. Poincaré-Verdier duality on the two-dimensional space $B$ tells us that

$$
\mathbb{R} \operatorname{Hom}\left(\mathbb{R} \Gamma\left(B, R^{i} \pi_{*} \mathbb{Z}\right), \mathbb{Z}\right) \cong \mathbb{R} \Gamma \mathbb{R} \mathcal{H o m}\left(R^{i} \pi_{*} \mathbb{Z}, \mathbb{Z}[2]\right) .
$$

Applying $H^{-j}$ to the complexes on the left- and right-hand side, we obtain

$$
H^{-j}\left(\mathbb{R} \operatorname{Hom}\left(\mathbb{R} \Gamma\left(B, R^{i} \pi_{*} \mathbb{Z}\right), \mathbb{Z}\right)\right) \cong \operatorname{Ext}^{2-j}\left(R^{i} \pi_{*} \mathbb{Z}, \mathbb{Z}\right) .
$$

To compute the left-hand side, use the spectral sequence with

$$
E_{2}^{p q}=\operatorname{Ext}^{p}\left(H^{-q}\left(B, R^{i} \pi_{*} \mathbb{Z}\right), \mathbb{Z}\right) \Rightarrow H^{n}\left(\mathbb{R} \operatorname{Hom}\left(\mathbb{R} \Gamma\left(B, R^{i} \pi_{*} \mathbb{Z}\right), \mathbb{Z}\right)\right),
$$

which yields exact sequences

$$
\begin{aligned}
0 \rightarrow \operatorname{Ext}^{1}\left(H^{j+1}\left(B, R^{i} \pi_{*} \mathbb{Z}\right), \mathbb{Z}\right) & \rightarrow H^{-j}\left(\mathbb{R} \operatorname{Hom}\left(\mathbb{R} \Gamma\left(B, R^{i} \pi_{*} \mathbb{Z}\right), \mathbb{Z}\right)\right) \\
& \rightarrow \operatorname{Hom}\left(H^{j}\left(B, R^{i} \pi_{*} \mathbb{Z}\right), \mathbb{Z}\right) \rightarrow 0 .
\end{aligned}
$$

To compute the right-hand side, we use the local-global Ext spectral sequence. Note that fibre-wise Poincaré duality for the fibres of $\pi$ over $B_{0}$ yields

$$
R^{p} \pi_{*} \mathbb{Z} \cong \mathcal{H o m}\left(R^{4-p} \pi_{*} \mathbb{Z}, \mathbb{Z}\right)
$$

on $B_{0}$. The fact that $i_{*} R^{p} \pi_{0 *} \mathbb{Z}=R^{p} \pi_{*} \mathbb{Z}$ on $B$ then tells us the same isomorphism holds on $B$. The higher sheaf $\mathcal{E} x t$ s can be computed locally on a contractible neighbourhood $U$ of a singular point $p$ : let $U_{0}=U \backslash\{p\}$ and let $i: U_{0} \rightarrow U$ be the inclusion. We compute $\operatorname{Ext}_{U}^{p}\left(R^{1} \pi_{*} \mathbb{Z}, \mathbb{Z}\right)$ using the description of monodromy; the other cases are similar. Because there are locally three independent monodromy invariant sections, we have an exact sequence on $U$

$$
0 \rightarrow \mathbb{Z}^{3} \rightarrow R^{1} \pi_{*} \mathbb{Z} \rightarrow i ! \mathbb{Z} \rightarrow 0 .
$$

Applying $\operatorname{Hom}_{U}(\cdot, \mathbb{Z})$ and the fact that $\operatorname{Ext}_{U}^{p}(i ! \mathbb{Z}, \mathbb{Z})=\operatorname{Ext}_{U_{0}}^{p}(\mathbb{Z}, \mathbb{Z})=H^{p}\left(U_{0}, \mathbb{Z}\right)$, we obtain $\operatorname{Ext}_{U}^{p}\left(R^{1} \pi_{*} \mathbb{Z}, \mathbb{Z}\right)=0$ for $p \geq 2$ and the exact sequence

$$
0 \rightarrow \mathbb{Z} \rightarrow \operatorname{Hom}_{U}\left(R^{1} \pi_{*} \mathbb{Z}, \mathbb{Z}\right) \rightarrow \mathbb{Z}^{3} \rightarrow H^{1}\left(U_{0}, \mathbb{Z}\right) \rightarrow \operatorname{Ext}_{U}^{1}\left(R^{1} \pi_{*} \mathbb{Z}, \mathbb{Z}\right) \rightarrow 0 .
$$

From the description of monodromy, it is then clear that the map $\mathbb{Z}^{3} \rightarrow H^{1}\left(U_{0}, \mathbb{Z}\right)=$ $\mathbb{Z}$ is surjective and $\operatorname{Ext}_{U}^{1}\left(R^{1} \pi_{*} \mathbb{Z}, \mathbb{Z}\right)=0$. Similarly, one finds $\mathcal{E} x t_{B}^{p}\left(R^{q} \pi_{*} \mathbb{Z}, \mathbb{Z}\right)=$ 0 for $p>0$ and all $q$. Thus $\operatorname{Ext}^{2-p}\left(R^{q} \pi_{*} \mathbb{Z}, \mathbb{Z}\right)=H^{2-p}\left(B, \mathcal{H o m}\left(R^{q} \pi_{*} \mathbb{Z}, \mathbb{Z}\right)\right)=$ $H^{2-p}\left(B, R^{4-q} \pi_{*} \mathbb{Z}\right)$.

Putting this all together, we see that if

$$
e^{p, q}=\operatorname{rank}_{\mathbb{Z}} H^{p}\left(B, R^{q} \pi_{*} \mathbb{Z}\right),
$$

then

$$
e^{p, q}=e^{2-p, 4-q}
$$


and that if

$$
T^{p, q}=\operatorname{Tors}\left(H^{p}\left(B, R^{q} \pi_{*} \mathbb{Z}\right)\right),
$$

then

$$
T^{p, q} \cong T^{3-p, 4-q} .
$$

Of course, $R^{q} \pi_{*} \mathbb{Z}$ is torsion free, so

$$
H^{0}\left(B, R^{q} \pi_{*} \mathbb{Z}\right)_{\text {tors }}=0
$$

anyway.

Next consider $H^{0}\left(B, R^{3} \pi_{*} \mathbb{Z}\right)=H^{0}\left(B_{0}, R^{3} \pi_{0 *} \mathbb{Z}\right)$. By [9], Corollary 4.1.2, $H^{0}\left(B_{0}, R^{3} \pi_{*} \mathbb{Z}\right)$ is a sub-Hodge structure of $H^{3}\left(\pi^{-1}(b), \mathbb{Z}\right)$ for any $b \in B_{0}$, and the Hodge structure on $H^{0}\left(B_{0}, R^{3} \pi_{*} \mathbb{Z}\right)$ is independent of $b$. However, if this group is non-zero, this implies $\pi^{-1}(b)$ contains a fixed non-trivial abelian subvariety for any $b \in B_{0}$. However, since the abelian surfaces appearing in $\pi: V_{8, y}^{1} \rightarrow B$ are general, this cannot happen. Thus $H^{0}\left(B, R^{3} \pi_{*} \mathbb{Z}\right)=0$. The entries in the $E_{2}$-term of the Leray spectral sequence of $\pi$ are now:

$$
\begin{array}{ccc}
\mathbb{Z} & 0 & \mathbb{Z} \\
0 & \mathbb{Z}^{e^{1,3}} \oplus T^{1,3} & \mathbb{Z}^{e^{2,3}} \oplus T^{2,3} \\
\mathbb{Z}^{e^{0,2}} & \mathbb{Z}^{e^{1,2}} \oplus T^{1,2} & \mathbb{Z}^{e^{2,2}} \oplus T^{2,2} \\
\mathbb{Z}^{e^{0,1}} & \mathbb{Z}^{e^{1,1}} \oplus T^{1,1} & T^{2,1} \\
\mathbb{Z} & 0 & \mathbb{Z}
\end{array}
$$

We note that because $\pi$ has a section, the boundary map $d: H^{0}\left(B, R^{4} \pi_{*} \mathbb{Z}\right) \rightarrow$ $H^{2}\left(B, R^{3} \pi_{*} \mathbb{Z}\right)$ is zero, so $H^{2}\left(B, R^{3} \pi_{*} \mathbb{Z}\right)$ injects into $H^{5}\left(V_{8, y}^{1}, \mathbb{Z}\right)=0$, so $H^{2}\left(B, R^{3} \pi_{*} \mathbb{Z}\right)=0$. Thus $e^{2,3}=e^{0,1}=0$, and $T^{2,3}=T^{1,1}=0$. Next, since $b_{2}\left(V_{8, y}^{1}\right)=2$ and $e^{0,2} \geq 1$, with $H$ generating a non-trivial class in $H^{0}\left(B, R^{2} \pi_{*} \mathbb{Z}\right)$, we see $e^{1,1}=e^{1,3}=0$ and $e^{0,2}=e^{2,2}=1$. So we now have:

$\begin{array}{ccc}\mathbb{Z} & 0 & \mathbb{Z} \\ 0 & T^{1,3} & 0 \\ \mathbb{Z} & \mathbb{Z}^{e^{1,2}} \oplus T^{1,2} & \mathbb{Z} \oplus T^{2,2} \\ 0 & 0 & T^{1,3} \\ \mathbb{Z} & 0 & \mathbb{Z}\end{array}$

Now $\pi: V_{8, y}^{1} \rightarrow B$ has a group of sections, once one of the 64 exceptional curves is chosen as a zero-section. This group of sections contains the group $(\mathbb{Z} / 8 \mathbb{Z})^{2}$, given by those 64 sections. Note that if $\pi^{-1}(b)=V / \Lambda$, where $V=\mathbb{C}^{2}$ and $\Lambda$ is a four-dimensional lattice, then $H^{3}\left(\pi^{-1}(b), \mathbb{Z}\right)=\Lambda$ and $H^{3}\left(\pi^{-1}(b), \mathbb{Z} / 8 \mathbb{Z}\right)=\Lambda / 8 \Lambda \cong$ $\frac{1}{8} \Lambda / \Lambda$. Thus $H^{3}\left(\pi^{-1}(b), \mathbb{Z} / 8 \mathbb{Z}\right)$ is canonically isomorphic to the set of 8 -torsion points on $\pi^{-1}(b)$, and $H^{0}\left(B, R^{3} \pi_{*} \mathbb{Z} / 8 \mathbb{Z}\right)=H^{0}\left(B_{0}, R^{3} \pi_{0 *} \mathbb{Z} / 8 \mathbb{Z}\right)$ is equal to the space of 8 -torsion sections, and hence contains the subgroup $(\mathbb{Z} / 8 \mathbb{Z})^{2}$. However, from the exact sequence

$$
0 \longrightarrow R^{3} \pi_{*} \mathbb{Z} \stackrel{\cdot 8}{\longrightarrow} R^{3} \pi_{*} \mathbb{Z} \longrightarrow R^{3} \pi_{*}(\mathbb{Z} / 8 \mathbb{Z}) \longrightarrow 0
$$

we see that $H^{0}\left(B, R^{3} \pi_{*} \mathbb{Z} / 8 \mathbb{Z}\right)=H^{1}\left(B, R^{3} \pi_{*} \mathbb{Z}\right)_{8}$, where the subscript denotes the part of the group killed by 8 . Thus $(\mathbb{Z} / 8 \mathbb{Z})^{2} \subseteq T^{1,3}$. This subgroup is naturally identified with $G$.

The Leray spectral sequence now gives us a filtration

$$
0=F^{0} \subseteq F^{1} \subseteq F^{2} \subseteq F^{3}=H^{4}\left(V_{8, y}^{1}, \mathbb{Z}\right)
$$


with $F^{1}=\mathbb{Z} \oplus T^{2,2}$ and $F^{2} / F^{1}=T^{1,3}$, so $H^{4}\left(V_{8, y}^{1}, \mathbb{Z}\right)$ contains the subgroup $F^{2}$ which is an extension

$$
0 \rightarrow \mathbb{Z} \oplus T^{2,2} \rightarrow F^{2} \rightarrow T^{1,3} \rightarrow 0 .
$$

We will show there is a map $\varphi: G \rightarrow F^{2}$ whose composition with the projection to $T^{1,3}$ is injective.

We will first have to show $T^{1,2}$ contains no two-torsion. Given $b \in B_{0}$, we have $H^{3}\left(\pi^{-1}(b), \mathbb{Z} / 2 \mathbb{Z}\right)=\Lambda / 2 \Lambda$, and we have the wedge product

$$
(\Lambda / 2 \Lambda) \times(\Lambda / 2 \Lambda) \rightarrow \bigwedge^{2}(\Lambda / 2 \Lambda)
$$

and

$$
(\Lambda / 2 \Lambda) \times\left(\bigwedge^{2}(\Lambda / 2 \Lambda)\right) \rightarrow \bigwedge^{3}(\Lambda / 2 \Lambda)
$$

Given linearly independent monodromy invariant elements $e_{1}, e_{2} \in \Lambda / 2 \Lambda$, we obtain a monodromy invariant element $e_{1} \wedge e_{2}$ of $\Lambda^{2}(\Lambda / 2 \Lambda)$. Suppose there is a twodimensional space of monodromy elements of $\Lambda^{2}(\Lambda / 2 \Lambda)$, say generated by $e_{1} \wedge$ $e_{2}$ and $f$. Then either $e_{1} \wedge f \neq 0$ or $e_{2} \wedge f \neq 0$ in $\Lambda^{3}(\Lambda / 2 \Lambda)$, and this would give a monodromy invariant element of $\Lambda^{3} \Lambda / 2 \Lambda^{3} \Lambda$. Thus we see that the fact that $H^{0}\left(B, R^{3} \pi_{*}(\mathbb{Z} / 2 \mathbb{Z})\right)$ contains two linearly independent elements implies that if $\operatorname{dim}_{\mathbb{Z} / 2 \mathbb{Z}} H^{0}\left(B, R^{2} \pi_{*}(\mathbb{Z} / 2 \mathbb{Z})\right) \geq 2$, then $H^{0}\left(B, R^{1} \pi_{*}(\mathbb{Z} / 2 \mathbb{Z})\right) \neq 0$, a contradiction. Thus $H^{1}\left(B, R^{2} \pi_{*} \mathbb{Z}\right)$ contains no 2 -torsion.

Now consider $\alpha \in G$, acting on $V_{8, y}^{1}$ by translation by an 8-torsion section. Then $\alpha^{*}: H^{4}\left(V_{8, y}^{1}, \mathbb{Z}\right) \rightarrow H^{4}\left(V_{8, y}^{1}, \mathbb{Z}\right)$ induces the identity on rational cohomology. Look at

$$
\log \alpha^{*}=\left(\alpha^{*}-I\right)-\frac{1}{2}\left(\alpha^{*}-I\right)^{2},
$$

where $I$ is the identity. This in fact acts on $H^{4}\left(V_{8, y}^{1}, \mathbb{Z}\right)$. Indeed $\alpha^{*}-I$ maps $H^{p}\left(B, R^{q} \pi_{*} \mathbb{Z}\right)$ to $H^{p+1}\left(B, R^{q-1} \pi_{*} \mathbb{Z}\right)$ because $\alpha^{*}-I$ acts trivially on the cohomology of a fibre of $\pi$. Thus $\left(\alpha^{*}-I\right)^{3}=0$, and since $T^{2,2} \cong T^{1,2}, H^{2}\left(B, R^{2} \pi_{*} \mathbb{Z}\right)_{\text {tors }}$ has no two-torsion, and so $\frac{1}{2}\left(\alpha^{*}-I\right)^{2}$ is well-defined.

If $e_{0}$ is the zero section of $\pi$, then we see the map $\varphi: G \rightarrow H^{4}\left(V_{8, y}^{1}, \mathbb{Z}\right)$ defined by $\alpha \mapsto \log \alpha^{*}\left(\left[e_{0}\right]\right)$ is a homomorphism:

$$
\begin{aligned}
\varphi(\alpha \circ \beta) & =\left(\log \alpha^{*} \circ \beta^{*}\right)\left[e_{0}\right] \\
& =\log \alpha^{*}\left(\left[e_{0}\right]\right)+\log \beta^{*}\left(\left[e_{0}\right]\right) \\
& =\varphi(\alpha)+\varphi(\beta) .
\end{aligned}
$$

The image of $\varphi$ is necessarily $G=(\mathbb{Z} / 8 \mathbb{Z})^{2}$, identified under projection to $T^{1,3}$ with the space of 8-torsion sections of $\pi$ contained in $T^{1,3}$. Thus, since $T^{2,2}$ has no twotorsion, we see $\varphi(\alpha)=\left(\alpha^{*}-I\right)\left(\left[e_{0}\right]\right)=[e]-\left[e_{0}\right]$, where $e=\alpha\left(e_{0}\right) \subseteq V_{8, y}^{1}$. So the subgroup of $H^{4}\left(V_{8, y}^{1}, \mathbb{Z}\right)$ generated by differences of exceptional curves is isomorphic to $G$. But as observed at the beginning of the proof, $\left\{[e]-\left[e_{0}\right]\right\}$ generates the torsion subgroup of $H^{4}\left(V_{8, y}^{1}, \mathbb{Z}\right)$, so $\operatorname{Tors}\left(H^{4}\left(V_{8, y}^{1}, \mathbb{Z}\right)\right)=(\mathbb{Z} / 8 \mathbb{Z})^{2}$.

Remark 1.5. Calculations performed in the second author's thesis ([17]) suggest that the mirror to $V_{8, y}^{1}$ has fundamental group $\mathbb{Z} / 8 \mathbb{Z}$ and Brauer group $\mathbb{Z} / 8 \mathbb{Z}$. This was derived from a conjectural topological torus fibration on $V_{8, y}^{1}$ and a calculation of these groups for the dual fibration. As yet, we do not have a rigorous construction 
of a mirror manifold, but one possible guess would be a quotient of $V_{8, y}^{1}$ by a subgroup $\mathbb{Z} / 8 \mathbb{Z} \subseteq G$.

\section{REFERENCES}

[1] Alexeev, V., and Nakamura, I., On Mumford's construction of degenerating abelian varieties, Tohoku Math. J. (2) 51 (1999), 399-420. MR.1707764 (2001g:14013)

[2] Artin, M., Néron models, in: Arithmetic geometry (Storrs, Conn., 1984), 213-230, Springer, New York, 1986. MR0861977

[3] Aspinwall, P., and Morrison, D., Stable singularities in string theory, with an appendix by M. Gross, Comm. Math. Phys. 178 (1996), 115-134. MR.1387944 (97d:32049)

[4] Batyrev, V., and Kreuzer, M., Integral cohomology and mirror symmetry for Calabi-Yau 3-folds, preprint, math.AG/0505432.

[5] Bayer, D., and Stillman, M., Macaulay: A system for computation in algebraic geometry and commutative algebra. Source and object code available for Unix and Macintosh computers. Contact the authors, or download from http://math.columbia.edu/ bayer/Macaulay.

[6] Bosch, S, Lütkebohmert, W., and Raynaud, M., Néron models. Ergebnisse der Mathematik und ihrer Grenzgebiete (3) 21. Springer-Verlag, Berlin, 1990. MR1045822 (91i:14034)

[7] Căldăraru, A., Derived categories of twisted sheaves on Calabi-Yau manifolds, Ph.D. thesis, Cornell University, 2000.

[8] Căldăraru, A., Derived categories of twisted sheaves on elliptic threefolds, J. Reine Angew. Math. 544 (2002), 161-179. MR 1887894 (2003a:14022)

[9] Deligne, P., Théorie de Hodge. II, Inst. Hautes Études Sci. Publ. Math. No. 40 (1971), 5-57. MR0498551 (58:16653a)

[10] Grayson, D., and Stillman, M., Macaulay 2: A computer program designed to support computations in algebraic geometry and computer algebra. Source and object code available from http://www.math.uiuc.edu/Macaulay2/.

[11] Gross, M., Special Lagrangian Fibrations I: Topology, in: Integrable Systems and Algebraic Geometry, (M.-H. Saito, Y. Shimizu and K. Ueno eds.), World Scientific 1998, 156-193. MR.1672120 (2000e:14066)

[12] Gross, M., Special Lagrangian Fibrations II: Geometry, in: Surveys in Differential Geometry, Somerville: MA, International Press 1999, 341-403. MR1772274 (2001j:53065)

[13] Gross, M., Topological Mirror Symmetry, Invent. Math. 144 (2001), 75-137. MR1821145 (2002c:14062)

[14] Gross, M., and Popescu, S., Equations of $(1, d)$-polarized abelian surfaces, Math. Ann. 310 (1998), 333-377. MR1602020 (99d:14046)

[15] Gross, M., and Popescu, S., Calabi-Yau threefolds and moduli of abelian surfaces. I Compositio Math. 127 (2001), 169-228. MR1845899 (2002f:14057)

[16] Hulek, K., and Weintraub, S., The principal degenerations of abelian surfaces and their polarisations, Math. Ann. 286 (1990), 281-307. MR1032935 (91e:14042)

[17] Pavanelli, S., Mirror symmetry for a two parameter family of Calabi-Yau three-folds with Euler characteristic 0, Ph.D thesis, University of Warwick, 2003.

[18] Shimura, G., Introduction to the arithmetic theory of automorphic functions, Publ. of the Math. Soc. of Japan, 11. Kanô Memorial Lectures, 1. Princeton University Press, Princeton, NJ, 1994. MR1291394 (95e:11048)

Department of Mathematics, University of California-San Diego, 9500 Gilman Drive, La Jolla, California 92093-0112

E-mail address: mgross@math.ucsd.edu

Nextra Investment Management SGR, Piazza Cadorna 3, 20123 Milano, Italy

E-mail address: simone_pavanelli@hotmail.com 